\title{
Lithospheric Deformation Associated with Two-Dimensional Strike-Slip Faulting
}

\author{
Sarva Jit Singh* and Sunita Rani \\ Department of Mathematics, Maharshi Dayanand University, \\ Rohtak-124001, India
}

\begin{abstract}
To model the coseismic and postseismic lithospheric deformations associated with faulting at a transform plate boundary, the problem of a long inclined strike-slip fault in a layer overlying a uniform half-space is discussed. Closed-form expressions for the static displacements and stresses are obtained when the two media are elastic. These expressions are used to study the effect of the source location and the dip of the fault on the surface deformation. The correspondence principle of linear viscoelasticity is used to obtain the quasi-static field when the layer is elastic and the half-space Maxwell viscoelastic. The coseismic field is modeled by the static response and the postseismic field is modeled by the quasi-static response minus the static response. The variation of the coseismic and postseismic surface displacement and shear stress with the distance from an inclined fault is studied for four source locations: a surface-breaking fault and three buried faults. Both the source location and the dip of the fault are found to influence the deformation field significantly. Contours of constant postseismic displacement and stresses on the distance-time grid are obtained. These contours are useful in examining the spatial and temporal dependence of the displacement and stress fields. It is found that while the nodal lines for the postseismic displacement are independent of time, the nodal lines for the postseismic shear stresses move away from the fault with time after the earthquake.
\end{abstract}

\section{Introduction}

The problem of forward modeling of transient quasi-static deformation has attracted the attention of several investigators. Rosenman and Singh (1973a, b) and Singh and Rosenman (1974) used the correspondence principle of linear viscoelasticity to obtain analytical expressions for the quasi-static displacements, strains and stresses due to a vertical rectangular fault in a viscoelastic half-space. Matsu'ura and Tanimoto (1980) and Iwasaki (1986) derived the corresponding results for an inclined fault. Rundle and Jackson (1977) developed an analytic approximation of Green's function for the displacements due to a vertical strike-slip point source in an elastic layer over a viscoelastic half-space. An approximation of Green's function is integrated analytically to obtain the displacements due to a finite rectangular strike-slip fault. Based on the

Received December 20, 1993; Accepted May 6, 1994

* To whom correspondence should be addressed. 
Thomson-Haskell formulation developed by Singh (1970), Rundle (1978) devised a method for computating the displacements due to arbitrary dislocation sources in an elastic layer over a viscoelastic half-space. Matsu'ura et al. (1981) and Iwasaki and Matsu'ura (1981) presented a method for the computation of quasi-static surface displacements, strains and tilts due to a dislocation source in a stratified elastic half-space with an intervenient Maxwellian layer. Iwasaki (1985) applied the same method to study the quasi-static deformation due to a dislocation source in a stratified medium consisting of elastic/viscoelastic layers lying over a viscoelastic half-space.

To construct a kinematic model for the earthquake cycle at convergent plate boundaries, Matsu'ura and Sato (1989) modeled the lithosphere-asthenosphere system by a stratified semi-infinite medium under gravity, consisting of an elastic surface layer, an intervening Maxwellian layer and an elastic substratum. The steady motion of plate convergence is represented by uniform slip at a constant rate on the upper boundary of the descending oceanic plate. Recently, a number of investigators have used dislocation sources in spherical earth models to study postseismic relaxation (see, e.g., Dragoni and Yuen, 1983; Dragoni et al., 1986; Pollitz, 1992; Pollitz and Sacks, 1992).

In the case of long faults, one is justified in using the two-dimensional (2-D) approximation which simplified the algebra to a great extent. Rybicki (1971) found a closed-form analytical solution for the problem of a long vertical strike-slip fault in a two-layer model of the Earth. Rybicki's solution has been used by Nur and Mavko (1974) and Cohen (1979), among others, to explain the postseismic surface deformation. While Nur and Mavko (1974) assumed the lithosphere-asthenosphere composite as an elastic layer overlying a standard linear viscoelastic half-space, Cohen (1979) assumed a standard linear viscoelastic layer overlying a Maxwell viscoelastic half-space. Savage and Prescott (1978) used the Nur-Mavko solution to construct a simple 2-D model of an earthquake cycle that takes place on a transform fault. Cohen (1982) used a finite element technique to calculate the postseismic deformation due to a long strike-slip fault in a multilayered model of the Earth consisting of an elastic upper lithosphere, a standard linear viscoelastic lower lithosphere, Maxwell viscoelastic asthenosphere and an elastic mesosphere. Bonafede et al. (1984) modeled a microplate as an elastic plate with two long strike-slip boundaries lying over a Maxwell viscoelastic asthenosphere.

In the present paper, Rybicki's solution is generalized to a fault of arbitrary dip. The correspondence principle of linear viscoelasticity is used to obtain the quasi-static field when the layer is elastic and the half-space is Maxwell viscoelastic. The static field is used to model the coseismic deformation following a strike-slip earthquake at a transform plate boundary and the quasi-static field minus the static field is used to model the postseismic deformation. The effect of the source location on the coseismic and postseismic deformations is studied by performing detailed numerical computations for four positions of the fault: one surface-breaking fault and three buried faults. It was found that the field caused by a surface-breaking fault is characteristically different from the field caused by a fault at depth. Graphs showing the effect of the dip angle on the variation of the coseismic and postseismic displacement and shear stress with distance from the fault are presented. Contour maps for the postseismic displacement and stresses on the distance-time grid are obtained. These maps display the spatial and temporal dependence of the postseismic deformation field. The main advantage 


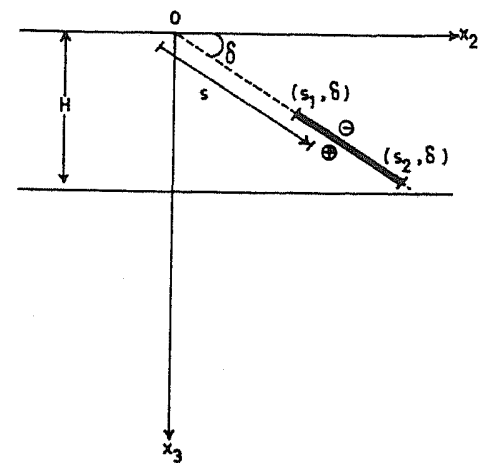

Fig. 1. Geometry of a long strike-slip fault situated in a layer of uniform thickness $H$ lying over a half-space. The displacement discontinuity on the fault is parallel to the $x_{1}$-axis. The sign $\oplus$ indicates displacement in the direction of the $x_{1}$-axis, the sign $\Theta$ in the opposite direction. The cartesian coordinates of a point on the fault are $\left(y_{2}, y_{3}\right)$ and its polar coordinates are $(s, \delta)$, where $\delta$ is the dip angle.

of considering the 2-D problem of a long strike-slip fault in an elastic layer over a viscoelastic half-space instead of the corresponding 3-D problem is that we are in a position to obtain a closed-form analytical solution of the problem. In the 3-D case, one is forced either to use approximate Green's functions (Rundle and Jackson, 1977) or to resort to numerical integration (Rundle, 1978).

We consider an Earth model consisting of a homogeneous, isotropic, elastic layer of thickness $H$ lying over a homogeneous, isotropic, Maxwell viscoelastic half-space (Fig. 1). We place the origin of a Cartesian coordinate system $\left(x_{1}, x_{2}, x_{3}\right)$ at the free surface and the $x_{3}$-axis is drawn into the medium. A long inclined strike-slip fault, with strike along the $x_{1}$-axis, is situated in the layer and $\left(y_{1}, y_{2}, y_{3}\right)$ is any point on the fault $\left(0 \leq y_{3}<H\right)$. Let $\mu_{1}$ and $\mu_{2}$ be the rigidities of the layer and of the half-space, respectively. The superscript (1) denotes quantities related to the layer and the superscript (2) denotes those related to the half-space. We first obtain the static response to a long inclined strike-slip fault in the corresponding elastic model. The correspondence principle of linear viscoelasticity is then used to obtain the quasi-static response.

\section{Elastic Solution}

Consider an antiplane strain problem for which the displacement components are of the form

$$
u=u\left(x_{2}, x_{3}\right), \quad v=w=0 .
$$

For zero body forces, the equilibrium equations reduce to

$$
\frac{\partial^{2} u}{\partial x_{2}{ }^{2}}+\frac{\partial^{2} u}{\partial x_{3}{ }^{2}}=0 \text {. }
$$

Vol. 42, No. 3, 1994 
The nonzero stresses are given by

$$
p_{12}=\mu \frac{\partial u}{\partial x_{2}}, \quad p_{13}=\mu \frac{\partial u}{\partial x_{3}} .
$$

The displacement field due to an inclined strike-slip line dislocation can be expressed in terms of the displacements due to a horizontal strike-slip line dislocation and a vertical strike-slip line dislocation in the form (Singh and Garg, 1985)

$$
u=\cos \delta u_{\mathrm{I}}-\sin \delta u_{\mathrm{II}},
$$

where $\delta$ is the dip angle (Fig. 1), $u_{\mathrm{I}}$ is the displacement for a horizontal strike-slip line dislocation and $u_{\mathrm{II}}$ is the displacement for a vertical strike-slip line dislocation. Using the results for the horizontal and vertical strike-slip line dislocations given by Garg and Sharma (1992) in Eq. (4), we obtain

$$
\begin{aligned}
u^{(1)}= & \frac{b d s}{2 \pi}\left\{\operatorname { c o s } \delta \left[\frac{x_{3}-y_{3}}{R^{2}}-\frac{x_{3}+y_{3}}{S^{2}}+\sum_{n=1}^{\infty} r^{n}\left\{\left(2 n H-x_{3}-y_{3}\right) \frac{1}{T^{2}}\right.\right.\right. \\
& \left.\left.+\left(2 n H+x_{3}-y_{3}\right) \frac{1}{V^{2}}-\left(2 n H-x_{3}+y_{3}\right) \frac{1}{U^{2}}-\left(2 n H+x_{3}+y_{3}\right) \frac{1}{W^{2}}\right\}\right] \\
& \left.-\sin \delta\left(x_{2}-y_{2}\right)\left[\frac{1}{R^{2}}+\frac{1}{S^{2}}+\sum_{n=1}^{\infty} r^{n}\left(\frac{1}{T^{2}}+\frac{1}{V^{2}}+\frac{1}{U^{2}}+\frac{1}{W^{2}}\right)\right]\right\}, \\
u^{(2)}= & \frac{b d s}{2 \pi}(1+r)\left\{\operatorname { c o s } \delta \left[\frac{x_{3}-y_{3}}{R^{2}}-\frac{x_{3}+y_{3}}{S^{2}}+\sum_{n=1}^{\infty} r^{n}\left\{\left(2 n H+x_{3}-y_{3}\right) \frac{1}{V^{2}}\right.\right.\right. \\
& \left.\left.-\left(2 n H+x_{3}+y_{3}\right) \frac{1}{W^{2}}\right\}\right]-\sin \delta\left(x_{2}-y_{2}\right)\left[\frac{1}{R^{2}}+\frac{1}{S^{2}}\right. \\
& \left.\left.+\sum_{n=1}^{\infty} r^{n}\left(\frac{1}{V^{2}}+\frac{1}{W^{2}}\right)\right]\right\},
\end{aligned}
$$

where

$$
\begin{aligned}
r & =\left(\mu_{1}-\mu_{2}\right) /\left(\mu_{1}+\mu_{2}\right), \\
R^{2} & =\left(x_{2}-y_{2}\right)^{2}+\left(x_{3}-y_{3}\right)^{2}, \\
S^{2} & =\left(x_{2}-y_{2}\right)^{2}+\left(x_{3}+y_{3}\right)^{2}, \\
T^{2} & =\left(x_{2}-y_{2}\right)^{2}+\left(2 n H-x_{3}-y_{3}\right)^{2}, \\
U^{2} & =\left(x_{2}-y_{2}\right)^{2}+\left(2 n H-x_{3}+y_{3}\right)^{2}, \\
V^{2} & =\left(x_{2}-y_{2}\right)^{2}+\left(2 n H+x_{3}-y_{3}\right)^{2}, \\
W^{2} & =\left(x_{2}-y_{2}\right)^{2}+\left(2 n H+x_{3}+y_{3}\right)^{2},
\end{aligned}
$$

$b$ is the slip and $d s$ is the width of the line dislocation.

Changing to polar coordinates (Fig. 1) 


$$
y_{2}=s \cos \delta, \quad y_{3}=s \sin \delta
$$

in Eqs. (5) and (6) and integrating over $s$ between the limits $\left(s_{1}, s_{2}\right)$, we obtain the following expressions for the displacements due to a long inclined strike-slip fault of finite width $s_{2}-s_{1}$ :

$$
\begin{aligned}
u^{(1)}= & \frac{1}{2 \pi}\left\{M_{0}\left[\tan ^{-1}\left(\frac{s-x_{2} \cos \delta-x_{3} \sin \delta}{x_{3} \cos \delta-x_{2} \sin \delta}\right)-\tan ^{-1}\left(\frac{s-x_{2} \cos \delta+x_{3} \sin \delta}{x_{3} \cos \delta+x_{2} \sin \delta}\right)\right]\right. \\
& +\sum_{n=1}^{\infty} M_{n}\left[\tan ^{-1}\left(\frac{s-x_{2} \cos \delta+x_{3} \sin \delta-2 n H \sin \delta}{2 n H \cos \delta-x_{3} \cos \delta-x_{2} \sin \delta}\right)\right. \\
& +\tan ^{-1}\left(\frac{s-x_{2} \cos \delta-x_{3} \sin \delta-2 n H \sin \delta}{2 n H \cos \delta+x_{3} \cos \delta-x_{2} \sin \delta}\right) \\
& -\tan ^{-1}\left(\frac{s-x_{2} \cos \delta-x_{3} \sin \delta+2 n H \sin \delta}{2 n H \cos \delta-x_{3} \cos \delta+x_{2} \sin \delta}\right) \\
& \left.\left.-\tan ^{-1}\left(\frac{s-x_{2} \cos \delta+x_{3} \sin \delta+2 n H \sin \delta}{2 n H \cos \delta+x_{3} \cos \delta+x_{2} \sin \delta}\right)\right]\right\}\left.\right|_{s_{1}} ^{s_{2}} \\
u^{(2)}= & \frac{1}{\pi}\left\{N_{0}\left[\tan ^{-1}\left(\frac{s-x_{2} \cos \delta-x_{3} \sin \delta}{x_{3} \cos \delta-x_{2} \sin \delta}\right)-\tan -1\left(\frac{s-x_{2} \cos \delta+x_{3} \sin \delta}{x_{3} \cos \delta+x_{2} \sin \delta}\right)\right]\right. \\
& +\sum_{n=1}^{\infty} N_{n}\left[\tan ^{-1}\left(\frac{s-x_{2} \cos \delta-x_{3} \sin \delta-2 n H \sin \delta}{2 n H \cos \delta+x_{3} \cos \delta-x_{2} \sin \delta}\right)\right. \\
& \left.\left.-\tan ^{-1}\left(\frac{s-x_{2} \cos \delta+x_{3} \sin \delta+2 n H \sin \delta}{2 n H \cos \delta+x_{3} \cos \delta+x_{2} \sin \delta}\right)\right]\right\}\left.\right|_{s_{1}} ^{s_{2}}
\end{aligned}
$$

where

$$
\begin{aligned}
& \left.f(s)\right|_{s_{1}} ^{s_{2}}=f\left(s_{2}\right)-f\left(s_{1}\right), \\
& M_{n}=b\left(\frac{\mu_{1}-\mu_{2}}{\mu_{1}+\mu_{2}}\right)^{n}, \\
& N_{n}=b \mu_{1} \frac{\left(\mu_{1}-\mu_{2}\right)^{n}}{\left(\mu_{1}+\mu_{2}\right)^{n+1}} .
\end{aligned}
$$

From Eqs. (3), (5), and (6), we get the following expressions for the stresses

$$
\begin{aligned}
p_{12}^{(1)} & =\frac{\mu_{1}}{2 \pi}\left\{M_{0}\left(-\frac{x_{3}-s \sin \delta}{R^{2}}+\frac{x_{3}+s \sin \delta}{S^{2}}\right)\right. \\
& +\sum_{n=1}^{\infty} M_{n}\left[\left(x_{3}+s \sin \delta-2 n H\right) \frac{1}{T^{2}}-\left(x_{3}-s \sin \delta+2 n H\right) \frac{1}{V^{2}}\right.
\end{aligned}
$$




$$
\begin{aligned}
- & \left.\left.\left(x_{3}-s \sin \delta-2 n H\right) \frac{1}{U^{2}}+\left(x_{3}+s \sin \delta+2 n H\right) \frac{1}{W^{2}}\right]\right\}\left.\right|_{s_{1}} ^{s_{2}} \\
p_{13}^{(1)}= & \left.\frac{\mu_{1}}{2 \pi}\left(x_{2}-s \cos \delta\right)\left[M_{0}\left(\frac{1}{R^{2}}-\frac{1}{S^{2}}\right)+\sum_{n=1}^{\infty} M_{n}\left(-\frac{1}{T^{2}}+\frac{1}{V^{2}}+\frac{1}{U^{2}}-\frac{1}{W^{2}}\right)\right]\right|_{s_{1}} ^{s_{2}} \\
p_{12}^{(2)}= & \frac{1}{\pi}\left\{P_{0}\left(-\frac{x_{3}-s \sin \delta}{R^{2}}+\frac{x_{3}+s \sin \delta}{S^{2}}\right)\right. \\
& \left.+\sum_{n=1}^{\infty} P_{n}\left[-\left(x_{3}-s \sin \delta+2 n H\right) \frac{1}{V^{2}}+\left(x_{3}+s \sin \delta+2 n H\right) \frac{1}{W^{2}}\right]\right\}\left.\right|_{s_{1}} ^{s_{2}} \\
p_{13}^{(2)}= & \left.\frac{1}{\pi}\left(x_{2}-s \cos \delta\right)\left[P_{0}\left(\frac{1}{R^{2}}-\frac{1}{S^{2}}\right)+\sum_{n=1}^{\infty} P_{n}\left(\frac{1}{V^{2}}-\frac{1}{W^{2}}\right)\right]\right|_{s_{1}} ^{s_{2}}
\end{aligned}
$$

where

$$
P_{n}=b \mu_{1} \mu_{2} \frac{\left(\mu_{1}-\mu_{2}\right)^{n}}{\left(\mu_{1}+\mu_{2}\right)^{n+1}}
$$

\section{Numerical Results for the Elastic Solution}

We wish to examine the effect of the dip angle and the location of the fault on the elastic deformation at the surface due to a strike-slip fault of finite width and infinite length embedded in an elastic layer lying over an elastic half-space. For this purpose, we assume $\mu_{1} / \mu_{2}=1 / 2$ and consider four different positions of the fault:

$$
\begin{array}{ll}
\text { Source I: } & s_{1}=0, \quad s_{2}=H / 4 \\
\text { Source II: } & s_{1}=H / 4, \quad s_{2}=H / 2 \\
\text { Source III: } & s_{1}=H / 2, \quad s_{2}=3 H / 4 \\
\text { Source IV: } & s_{1}=3 H / 4, \quad s_{2}=H .
\end{array}
$$

Figure 2(a) shows the variation of the dimensionless parallel surface displacement $(u / b)$ with the distance from the fault strike $\left(x_{2}\right)$ for $\delta=30^{\circ}$. We notice that the displacement field due to the surface-breaking fault (Source I) is altogether different from the field due to the other three sources. For Source I, $u$ is discontinuous at $x_{2}=0$; for the other three sources, $u$ is continuous at $x_{2}=0$. The variation of $u$ with $x_{2}$ for $\delta=60^{\circ}$ is shown in Fig. 2(b). Figure 2(c) is for $\delta=90^{\circ}$. In this case, $u$ is antisymmetric about the origin. For Source $\mathrm{I}, u$ is discontinuous at $x_{2}=0$. For the other three sources, $u$ is continuous at $x_{2}=0$ and vanishes at that point.

Figure 3(a) illustrates the variation of the dimensionless surface shear stress $P_{12}$ $\left[=p_{12} /\left(\mu_{1} b / H\right)\right]$ with the distance from the fault dipping at $30^{\circ} . P_{12}$ is positive for Source I. Figure 3(b) is for $\delta=60^{\circ}$. The behavior of $P_{12}$ for a vertical strike-slip fault $\left(\delta=90^{\circ}\right)$ is shown in Fig. 3(c). In this case, $P_{12}$ is completely symmetric about the origin. 
(a)

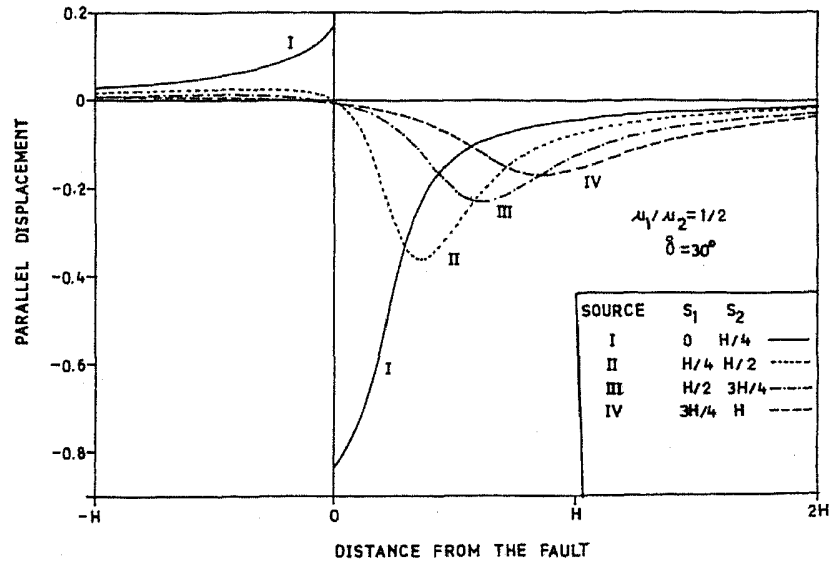

(b)

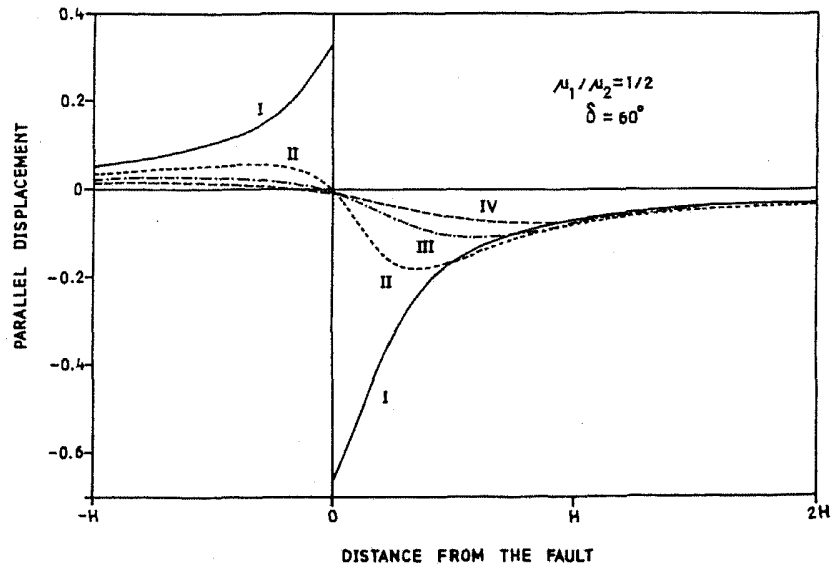

(c)

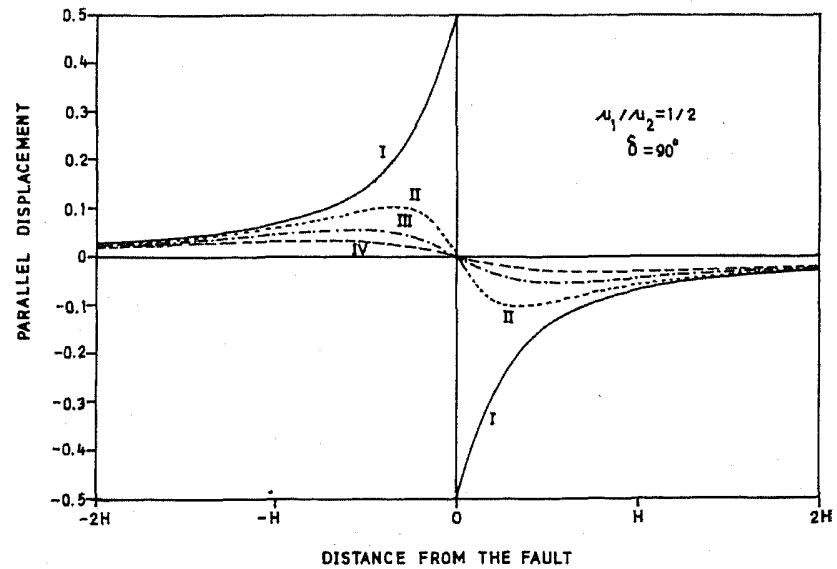

Fig. 2. Variation of the dimensionless parallel displacement $(u / b)$ at the surface with the distance from the fault $\left(x_{2}\right)$ for $\mu_{1} / \mu_{2}=1 / 2$ and (a) $\delta=30^{\circ}$, (b) $\delta=60^{\circ}$, (c) $\delta=90^{\circ}$.

Vol. 42, No. 3, 1994 
(a)

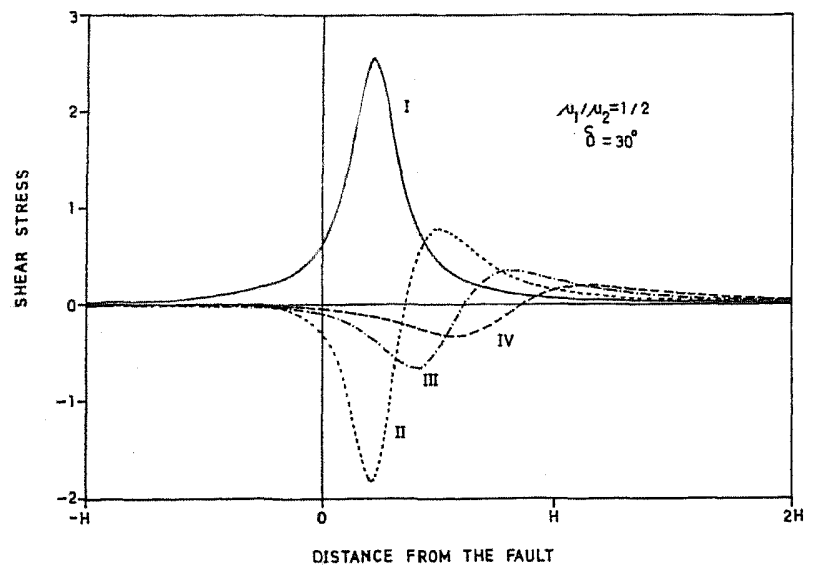

(b)

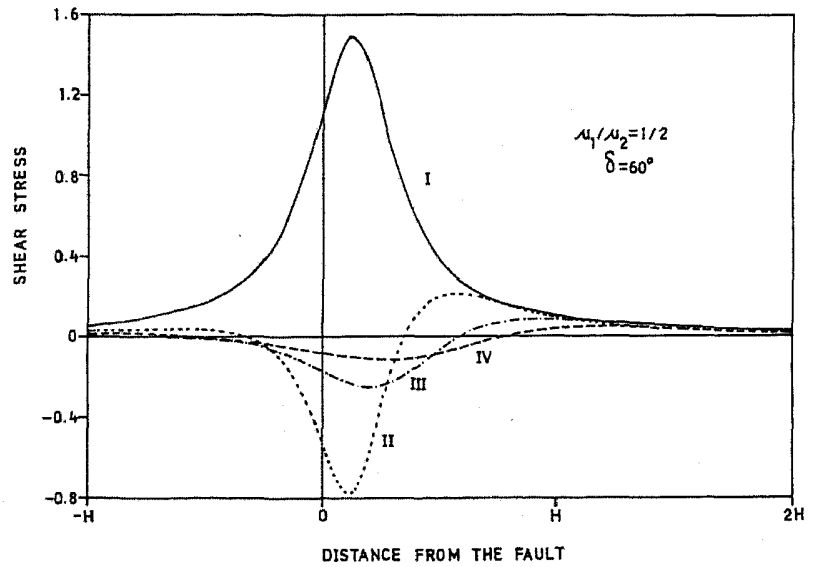

(c)

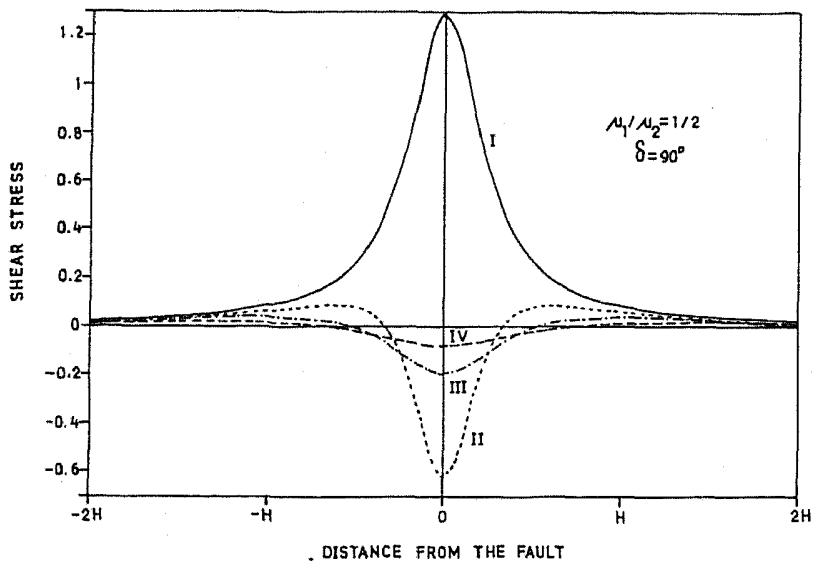

Fig. 3. Variation of the dimensionless shear stress $P_{12}=p_{12} /\left(\mu_{1} b / H\right)$ at the surface with the distance from the fault $\left(x_{2}\right)$ for $\mu_{1} / \mu_{2}=1 / 2$ and (a) $\delta=30^{\circ}$, (b) $\delta=60^{\circ}$, (c) $\delta=90^{\circ}$. 


\section{Viscoelastic Solution}

We use the correspondence principle of linear viscoelasticity to obtain the quasi-static deformation for a model consisting of an elastic layer lying over a Maxwell viscoelastic half-space. For the half-space, the constitutive equations may be written in the form (Lee, 1955)

$$
\bar{p}_{12}^{(2)}=2 \mu_{2}^{*} \bar{e}_{12}^{(2)}, \quad \bar{p}_{13}^{(2)}=2 \mu_{2}^{*} \bar{e}_{13}^{(2)},
$$

where an overbar indicates a Laplace transform, $\mu_{2}^{*}=p \mu_{2} /\left(p+\tau^{-1}\right)$ is the transform rigidity, $\tau=\eta / 2 \mu_{2}$ is the relaxation time, $\eta$ is the viscosity and $p$ is the Laplace transform variable. The time-dependence of the dislocation source is taken to be a unit step function, i.e., $b(t)=b_{0} H(t)$, where $b_{0}$ and $H(t)$ are, respectively, the magnitude of the slip (dislocation) and the Heaviside unit step function. Therefore, $\bar{b}=b_{0} / p$.

To obtain the Laplace transformed viscoelastic solution, it is only necessary to replace $\mu_{2}$ and $b$ by $\mu_{2}^{*}$ and $\bar{b}$, respectively, in the corresponding elastic solution. From Eqs. (9)-(16), we notice that $\mu_{2}$ and $b$ occur only through $M_{n}, N_{n}$, and $P_{n}$. Therefore, the Laplace-transformed solution of the viscoelastic problem is obtained from Eqs. (9), (10), and (12)-(15) on replacing $M_{n}, N_{n}$, and $P_{n}$ by $\bar{M}_{n}, \bar{N}_{n}$, and $\bar{P}_{n}$, respectively, where, from Eqs. (11) and (16),

$$
\begin{aligned}
& \bar{M}_{n}=\frac{b_{0}}{p}\left(\frac{p B+A}{p+A}\right)^{n} \\
& \bar{N}_{n}=\frac{b_{0}}{C p}\left(p+\tau^{-1}\right) \frac{(p B+A)^{n}}{(p+A)^{n+1}} \\
& \bar{P}_{n}=\frac{b_{0}(p B+A)^{n}}{D(p+A)^{n+1}}
\end{aligned}
$$

and

$$
\begin{array}{ll}
A \frac{\mu_{1}}{\tau\left(\mu_{1}+\mu_{2}\right)}, & B=\frac{\mu_{1}-\mu_{2}}{\mu_{1}+\mu_{2}}, \\
C=\frac{\mu_{1}+\mu_{2}}{\mu_{1}}, & D=\frac{\mu_{1}+\mu_{2}}{\mu_{1} \mu_{2}} .
\end{array}
$$

To find the inverse Laplace transforms of $\bar{M}_{n}, \bar{N}_{n}$, and $\bar{P}_{n}$, we used the integral transform tables of Erdélyi (1954). We obtain

$$
\begin{aligned}
& L^{-1}\left[\bar{M}_{0}\right]=b_{0}, \\
& L^{-1}\left[\bar{M}_{n}\right]=b_{0}\left[1+\exp (-A t) \sum_{m=1}^{n} \frac{F_{2 m}(-A)}{(n-m) !(m-1) !} t^{n-m}\right], \\
& L^{-1}\left[\bar{N}_{0}\right]=\frac{b_{0}}{C}\left[\frac{1}{\tau A}+\left(1-\frac{1}{\tau A}\right) \exp (-A t)\right]
\end{aligned}
$$

Vol. 42, No. 3, 1994 


$$
\begin{aligned}
L^{-1}\left[N_{n}\right]= & \frac{b_{0}}{C}\left[\frac{1}{\tau A}+\left(1-\frac{1}{\tau A}\right) \exp (-A t) \frac{(A t)^{n}(1-B)^{n}}{n !}\right. \\
& \left.+\sum_{m=1}^{n} \frac{t^{n-m}}{(n-m) ! m !} \exp (-A t) \phi_{2 m}(-A)\right] \\
L^{-1}\left[\bar{P}_{0}\right]= & \frac{b_{0}}{D} \exp (-A t) \\
L^{-1}\left[\bar{P}_{n}\right]= & \frac{b_{0}}{D} \exp (-A t)\left[(A t)^{n} \frac{(1-B)^{n}}{n !}+\sum_{m=1}^{n}(A t)^{n-m} B^{m} \frac{(1-B)^{n-m}}{[(n-m) !]^{2}} \cdot \frac{n !}{m !}\right],
\end{aligned}
$$

where $t>0, n>0$, and

$$
\begin{aligned}
& F_{2 m}(p)=\frac{\mathrm{d}^{m-1}}{\mathrm{~d} p^{m-1}}\left[\frac{(p B+A)^{n}}{p}\right] \\
& \phi_{2 m}(p)=\frac{\mathrm{d}^{m}}{\mathrm{~d} p^{m}}\left[(p B+A)^{n} \frac{\left(p+\tau^{-1}\right)}{p}\right] .
\end{aligned}
$$

\section{Numerical Results for the Viscoelastic Solution}

We consider the particular case when the rigidities $\mu_{1}$ and $\mu_{2}$ are equal, i.e., $\mu_{1}=\mu_{2}=\mu$ (say). Equation (19) shows that, for this particular case,

$$
A=1 / 2 \tau, \quad B=0, \quad C=2, \quad D=2 / \mu .
$$

Equations (21) and (22) yield

$$
\begin{aligned}
& F_{2 m}(-A)=-\frac{(m-1) !}{(2 \tau)^{n-m}}, \\
& \phi_{2 m}(-A)=-2 \frac{m !}{(2 \tau)^{n-m}} .
\end{aligned}
$$

We wish to compute the coseismic and postseismic surface displacement and shear stress. The coseismic field is modeled by the static response. The postseismic field is obtained by subtracting the static response from the quasi-static response. Figure 4(a) shows the variation of the coseismic and postseismic parallel displacement $(u)$ at the surface in units of the slip $b$ for a surface-breaking fault (Source I) dipping at $45^{\circ}$. The coseismic displacement is discontinuous at $x_{2}=0$ while the postseismic displacement is continuous at $x_{2}=0$ and vanishes at $x_{2}=0.17 \mathrm{H}$. Figure $4(\mathrm{~b})-(\mathrm{d})$ is for Sources II, III, and IV, respectively. The coseismic displacement vanishes at $x_{2}=0$ and the postseismic displacement vanishes at $x_{2}=0.51 \mathrm{H}, 0.82 \mathrm{H}$, and $1.09 \mathrm{H}$ for Sources II, III, and IV, respectively. We note that the distance from the fault of the point on the surface of the Earth at which the postseismic surface displacement vanishes is independent of time. It depends, however, on the source location and dip angle. Figure 

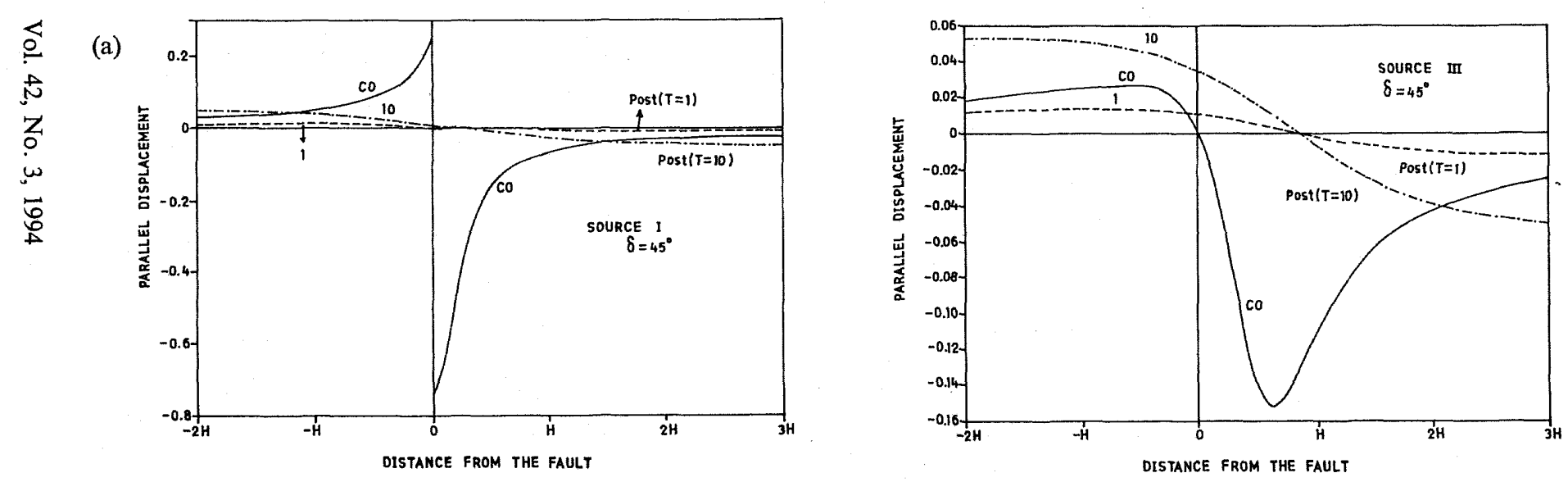

(b)
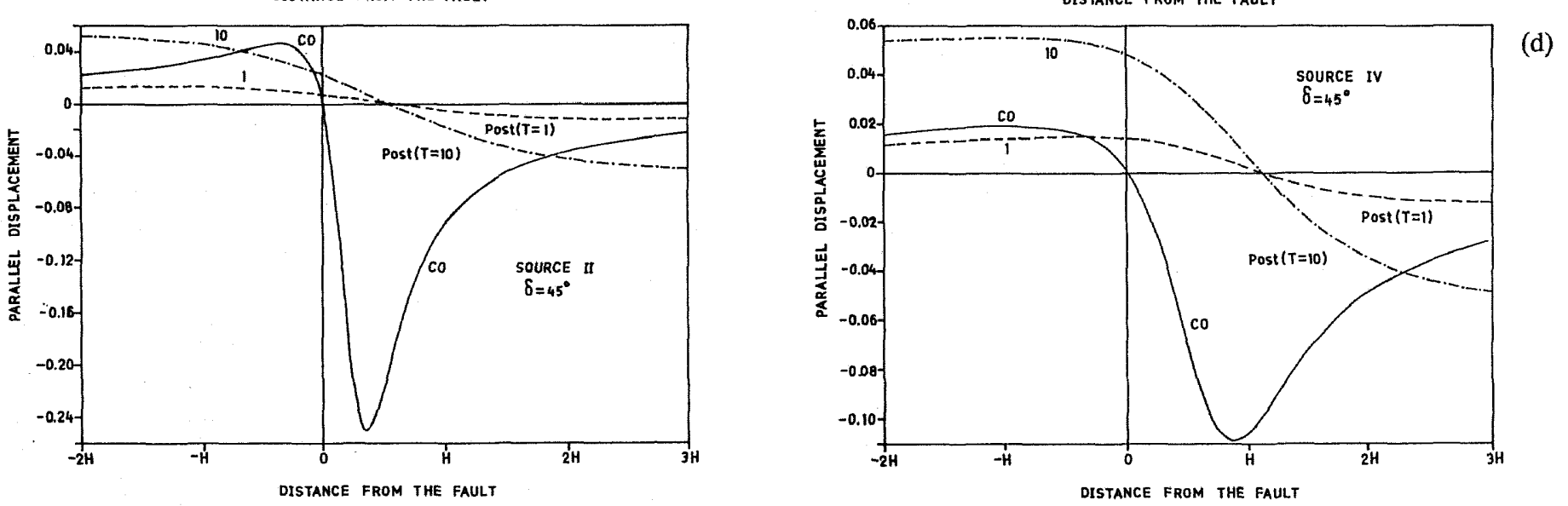

Fig. 4. Variation of the coseismic and postseismic parallel surface displacement in units of the slip $b$ with the distance from the fault $\left(x_{2}\right)$ assuming $\delta=45^{\circ}, \mu_{1}=\mu_{2}=\mu$, for (a) Source I, (b) Source II, (c) Source III, (d) Source IV. $\mathrm{CO}$ indicates coseismic (static) displacement. POST indicates postseismic (quasi-static minus static) displacement and is shown for two values of the dimensionless time $T=t / \tau$, where $\tau$ is the relaxation time. 
(a)

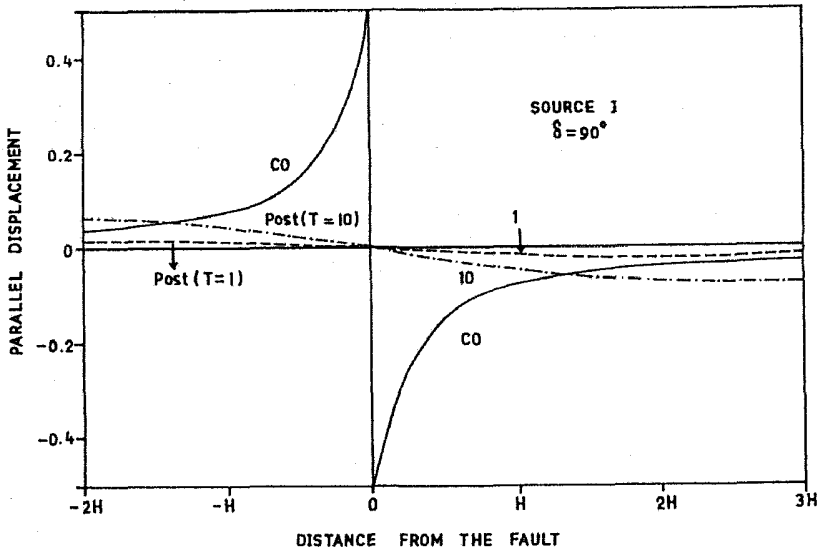

(b)

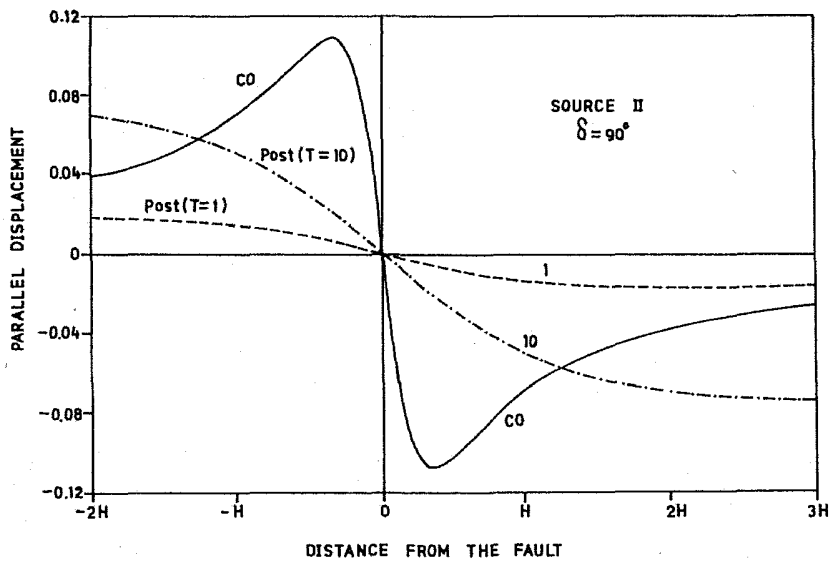

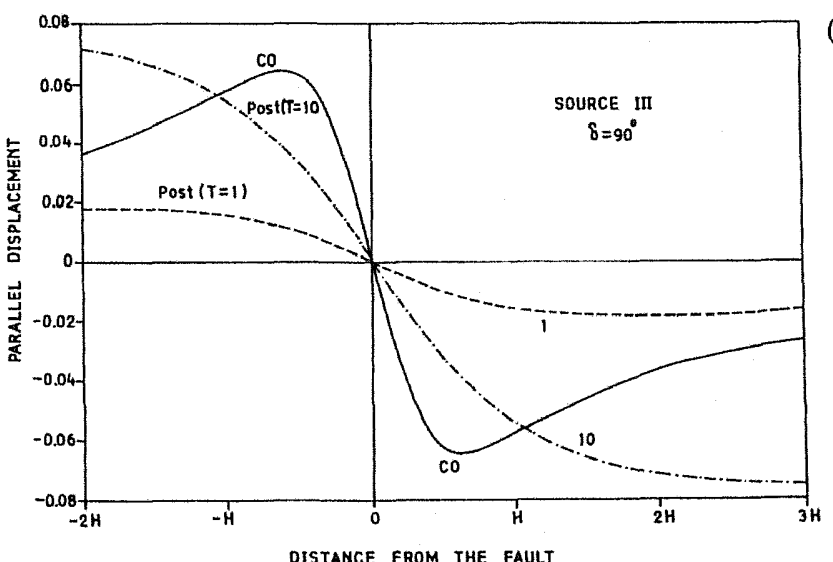

(c) $\stackrel{\sim}{\infty}$

\section{in}

电

骂

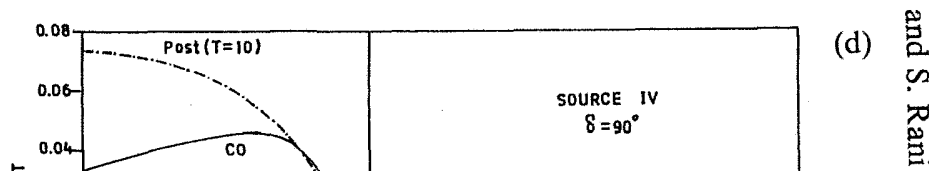

Fig. 5. Variation of the coseismic and postseismic parallel surface displacement in units of the slip $b$ with the distance from the fault ( $\left.x_{2}\right)$, assuming $\mu_{1}=\mu_{2}=\mu, \delta=90^{\circ}$ for (a) Source I, (b) Source II, (c) Source III, (d) Source IV. 

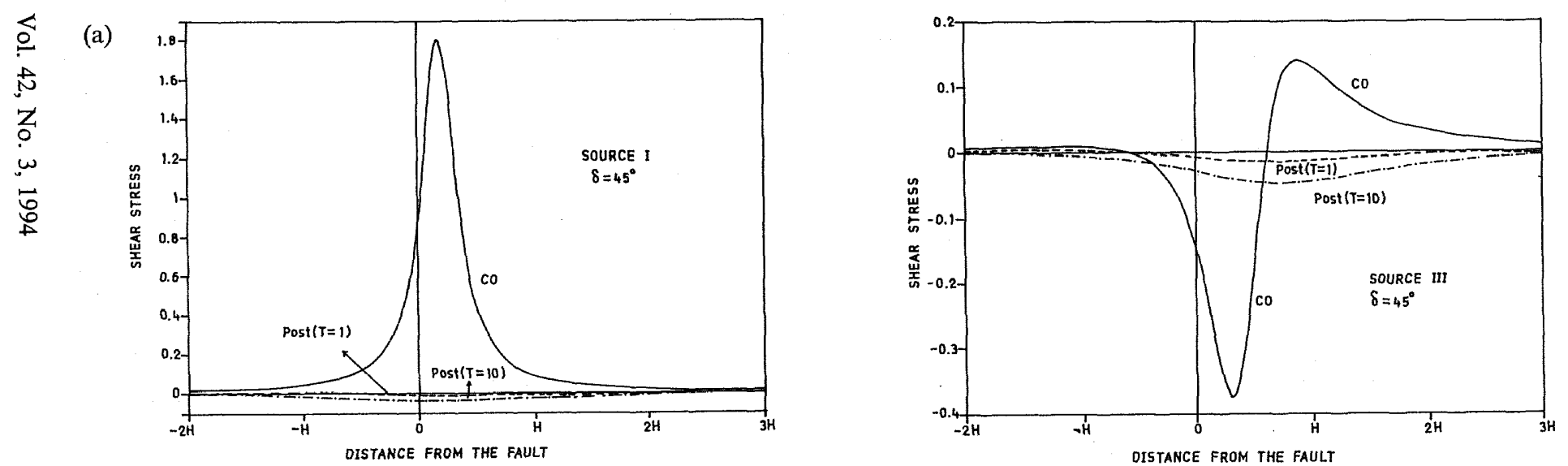

(c)

(b)
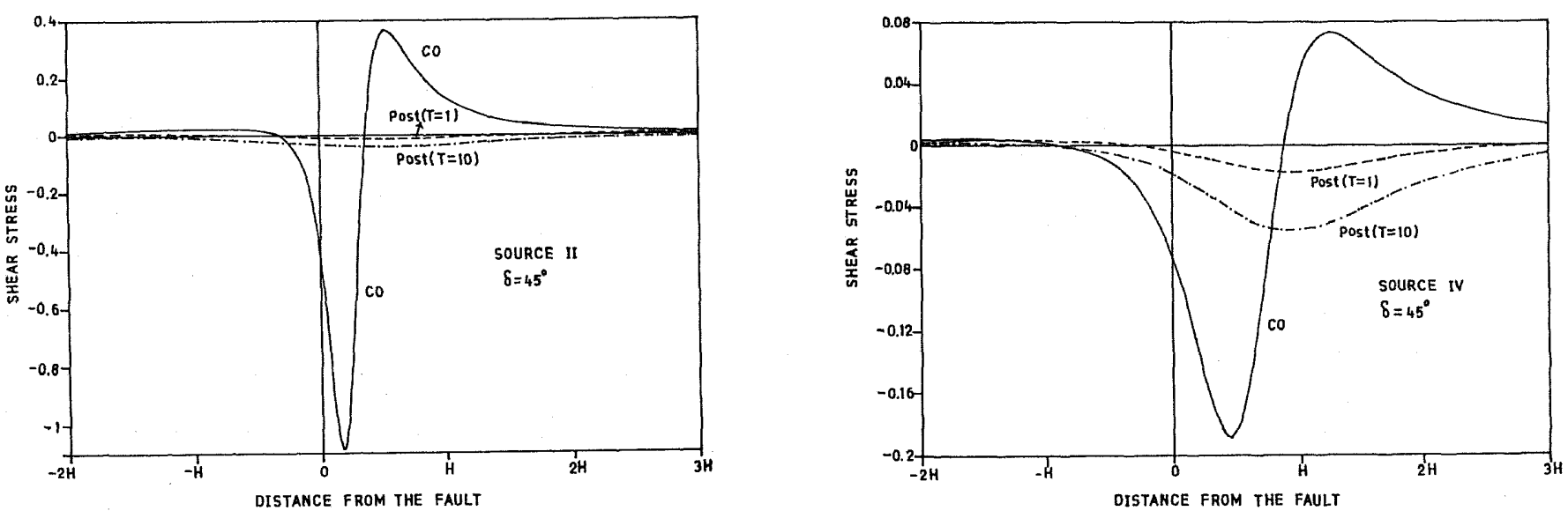

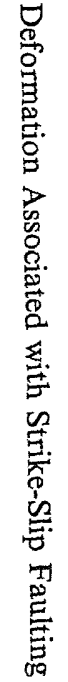

Fig. 6. Variation of the coseismic and postseismic shear stress $P_{12}=p_{12} /(\mu b / H)$ at the surface with the distance from the fault $\left(x_{2}\right)$, assuming $\mu_{1}=\mu_{2}=\mu, \delta=45^{\circ}$ for (a) Source I, (b) Source II, (c) Source III, (d) Source IV. 
(a)

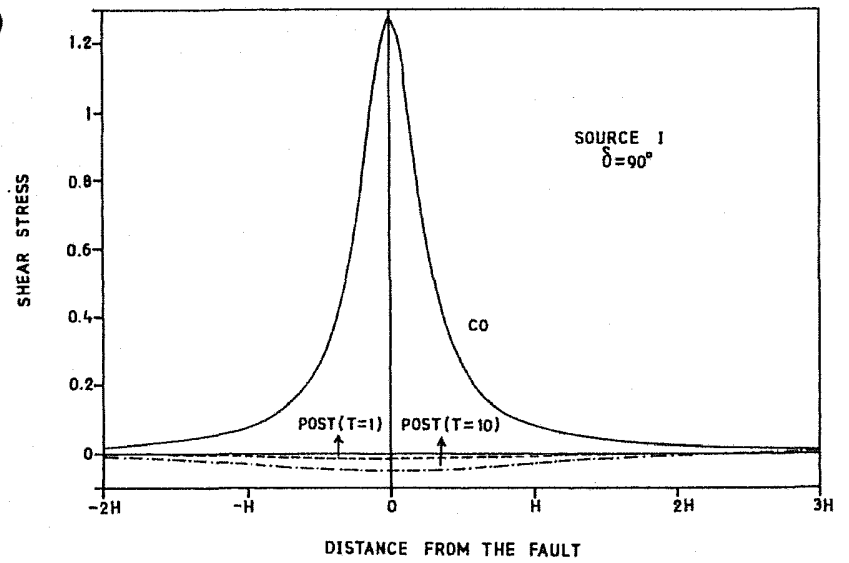

(b)

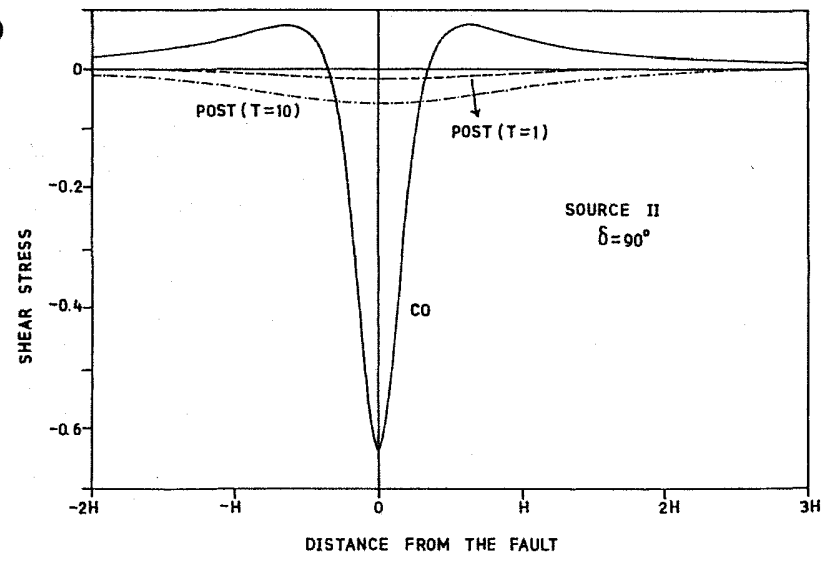

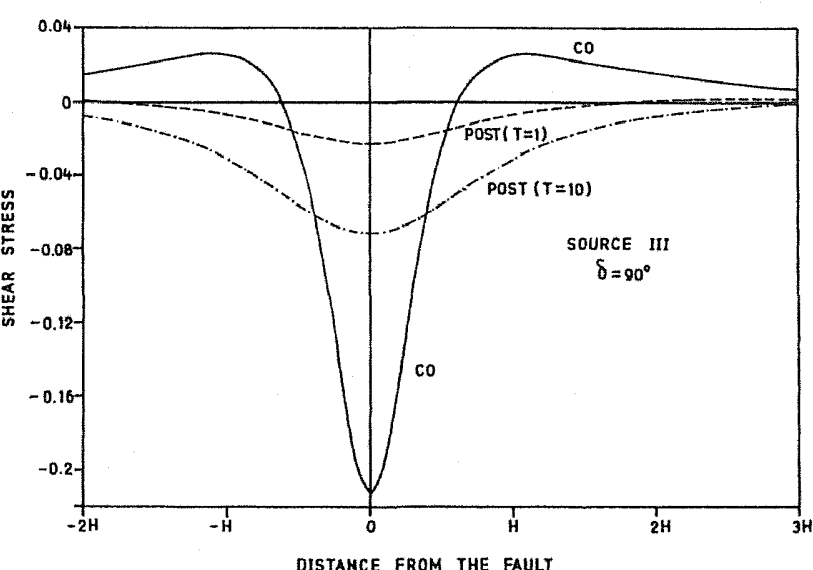

(c)

$\frac{N}{O}$

0
0
0
0
0
0
0
0
0
0
0
0
0
0
0

Fig. 7. Variation of the coseismic and postseismic shear stress $P_{12}$ at the surface with the distance from the fault ( $\left.x_{2}\right)$ assuming $\mu_{1}=\mu_{2}=\mu, \delta=90^{\circ}$ for (a) Source I, (b) Source II, (c) Source III, (d) Source IV. 

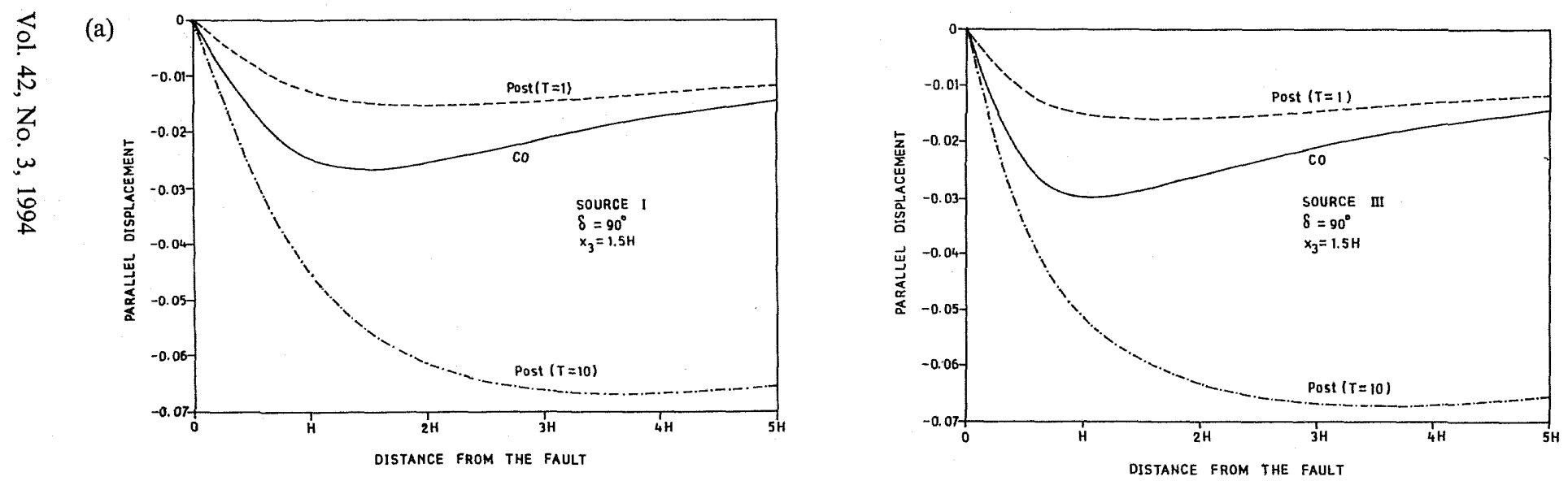

(c)

(b)

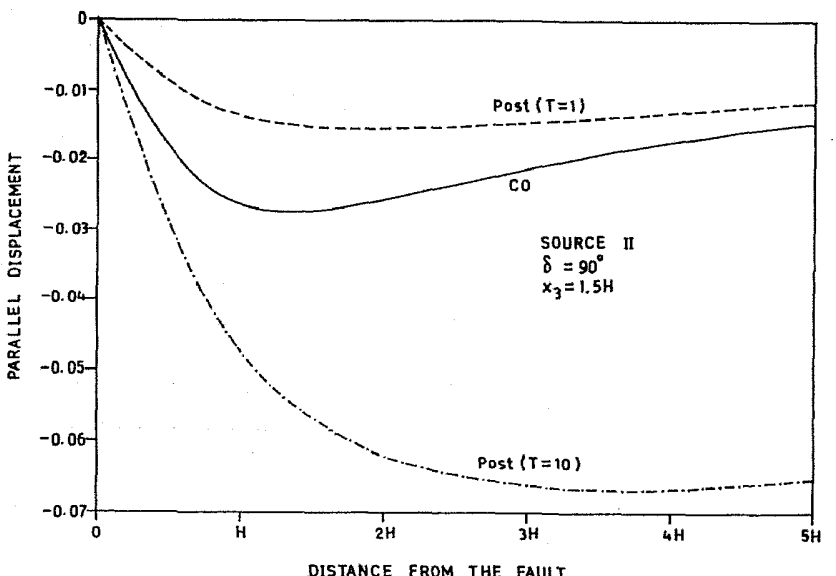

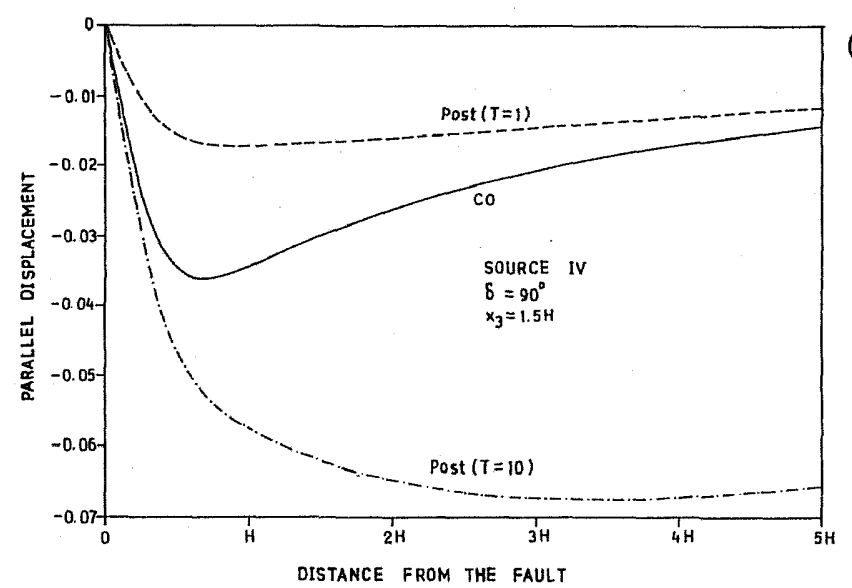

(d)

Fig. 8. Variation of the coseismic and postseismic parallel subsurface displacement in units of the slip $b$ with the distance from the fault $\left(x_{2}\right)$ assuming $\delta=90^{\circ}, \mu_{1}=\mu_{2}=\mu, x_{3}=1.5 H$, for (a) Source I, (b) Source II, (c) Source III, (d) Source IV. 
(a)

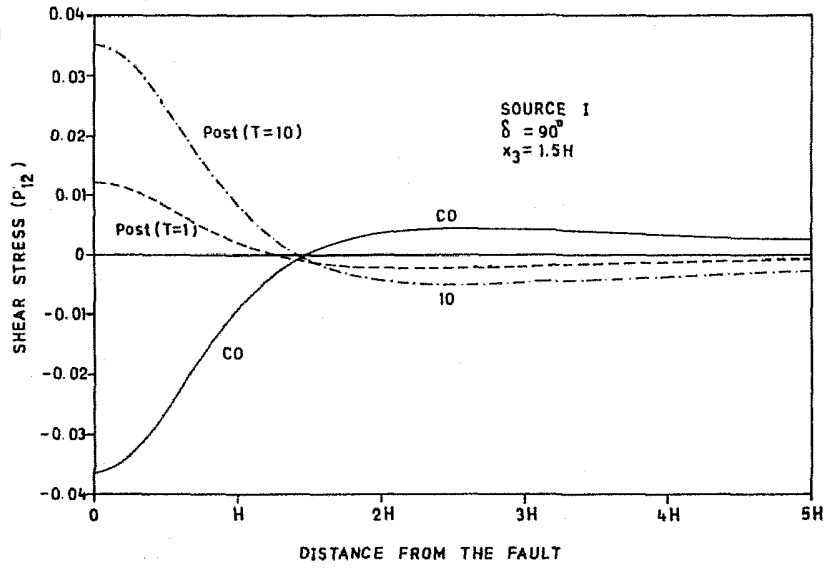

(b)

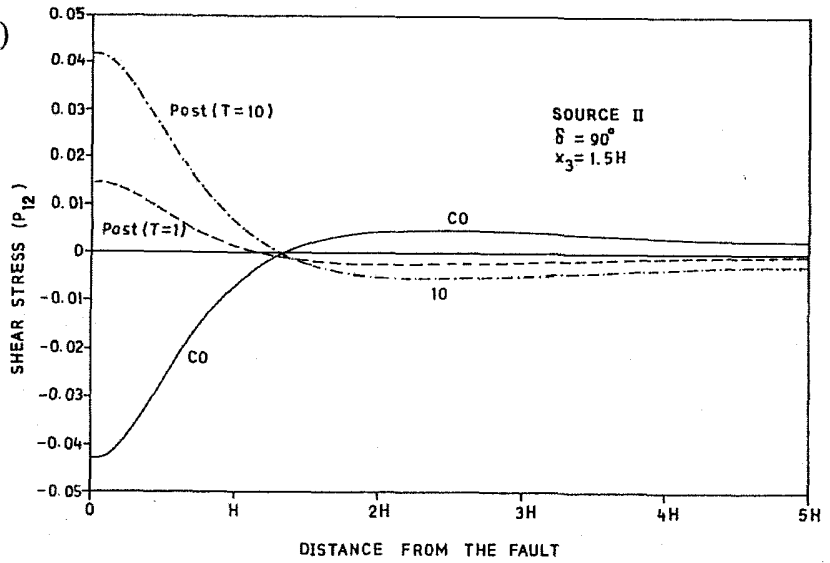
Source I, (b) Source II, (c) Source III, (d) Source IV.
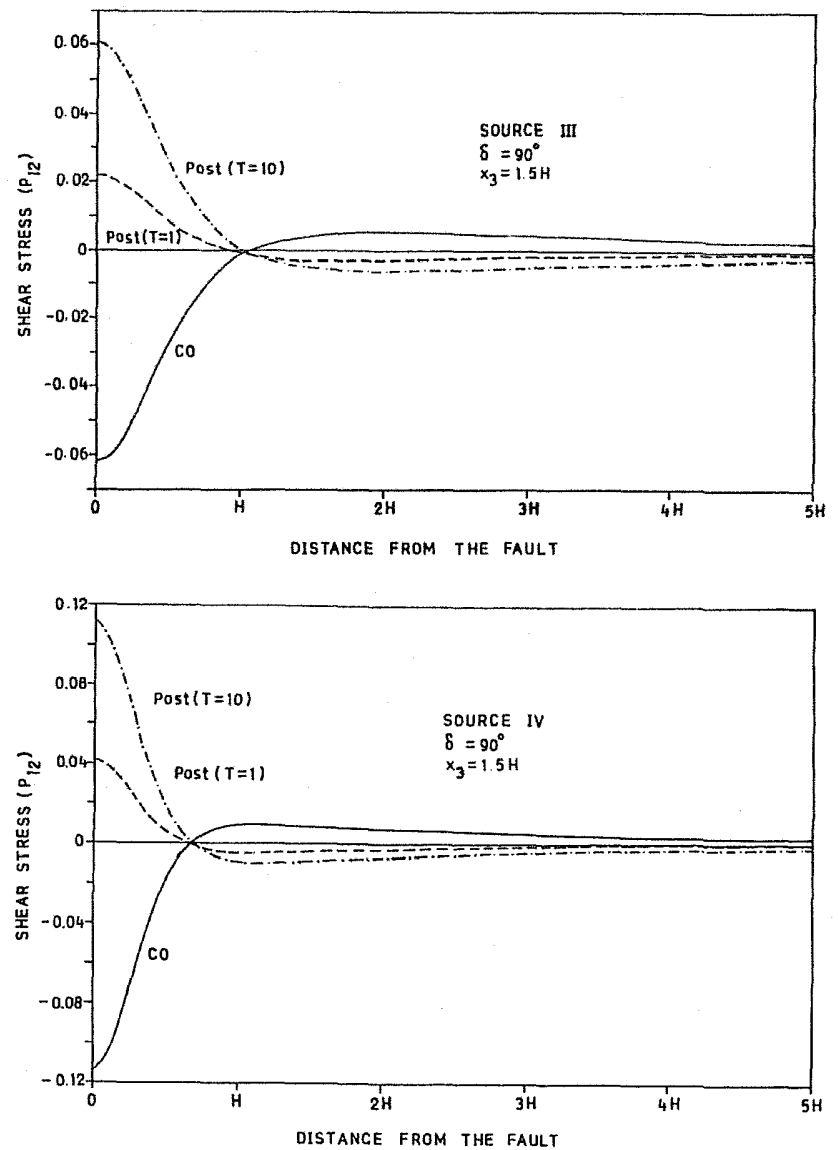

(c) $\stackrel{N}{N}$

Fig. 9. Variation of the coseismic and postseismic shear stress $P_{12}=p_{12} /(\mu b / H)$ in the viscoelastic half-space below the elastic surface layer with the distance from the fault, assuming $\mu_{1}=\mu_{2}=\mu, \delta=90^{\circ}, x_{3}=1.5 H$, for (a) 

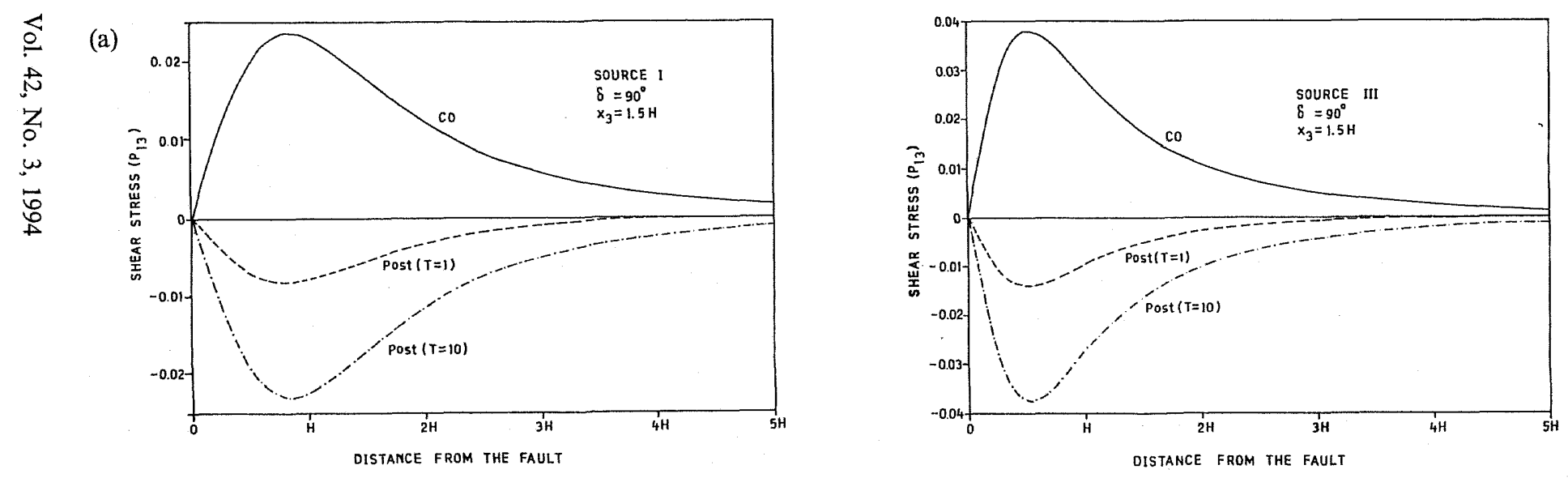

(c)
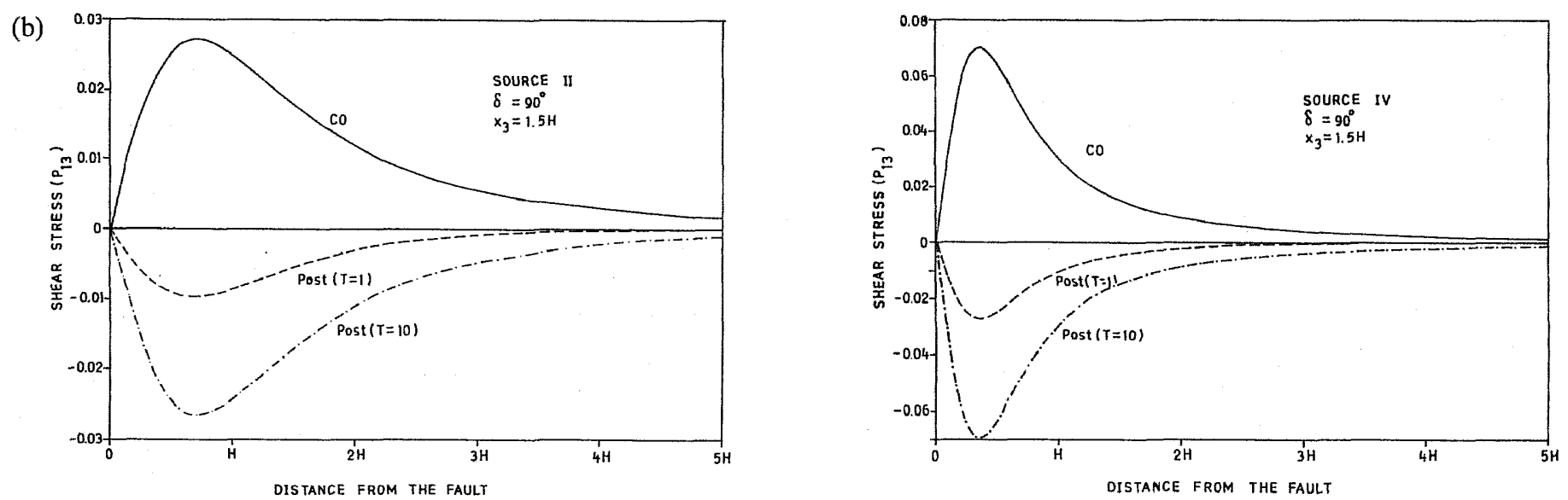

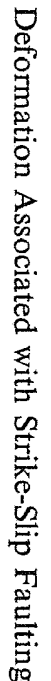

Fig. 10. Variation of the coseismic and postseismic shear stress $P_{13}=p_{13} /(\mu b / H)$ in the viscoelastic half-space below the elastic surface layer, for (a) Source I, (b) Source II, (c) Source III, (d) Source IV. 


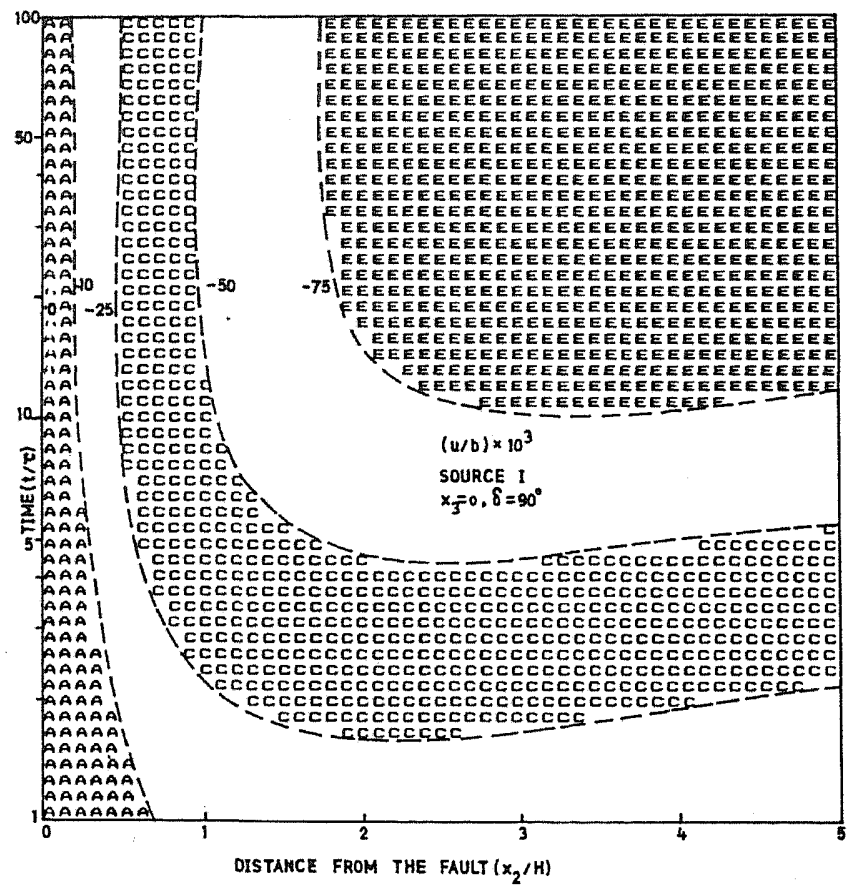

(a)

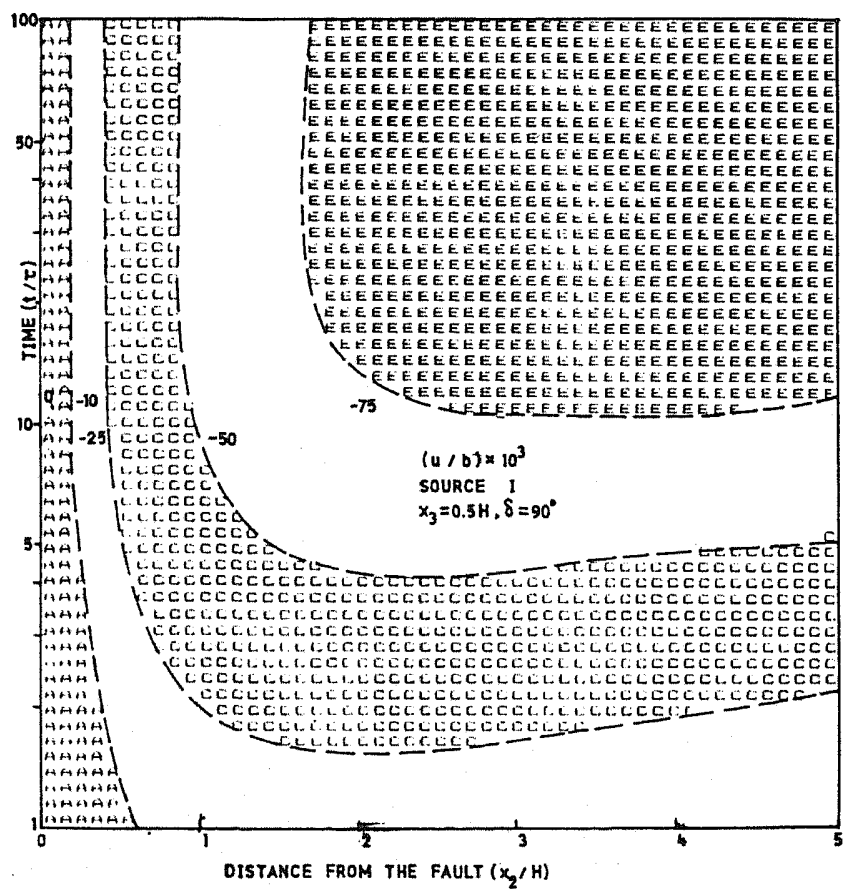

(b) 


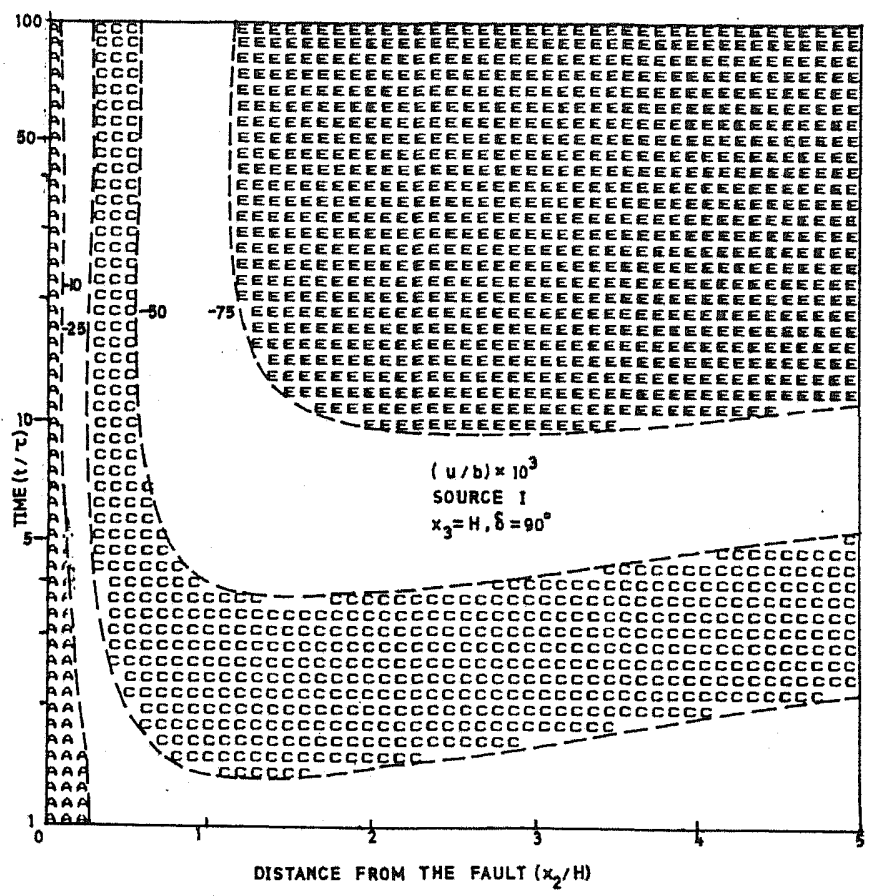

(c)

Fig. 11. Contours of constant postseismic parallel displacement on the distance from the fault-time after the earthquake grid for a surface-breaking vertical fault of depth $H / 4$, assuming $\mu_{1}=\mu_{2}=\mu$, for (a) $x_{3}=0$, (b) $x_{3}=H / 2$, (c) $x_{3}=H$.

5(a)-(d) illustrates the variation of the coseismic and postseismic displacements with the distance from the fault for a vertical fault. The displacement field is antisymmetric about the origin. The coseismic and postseismic displacements are of the same sign. The coseismic displacement is discontinuous at the origin for Source $I$ and is continuous for the other three sources at $x_{2}=0$ and vanishes at that point. The postseismic displacement is continuous at $x_{2}=0$ for Source I also and vanishes there.

Figure 6(a) shows the fall-off of the dimensionless coseismic and postseismic shear stress $P_{12}=p_{12} /(\mu b / H)$ at the surface for $\delta=45^{\circ}$. The coseismic stress varies significantly in the range $-H \leq x_{2}<H$ and attains its maximum at $x_{2}=0.18 H$. Figure 6(b)-(d) is for Sources II, III, and IV, respectively. The points on the surface of the Earth at which the postseismic shear stress vanishes move away from the fault with time. Figure 7(a)-(d) gives the variation of the dimensionless coseismic and postseismic shear stress $P_{12}$ for a vertical fault. $P_{12}$ is symmetric about the origin.

Since the stress relaxation process in the viscoelastic substratum is significantly different from that in the elastic surface layer, we have computed the coseismic and postseismic field in the underlying half-space as well at a depth of $3 H / 2$. Figure 8(a)-(d) depicts the variation of the parallel displacement with the distance from the fault. Figure 9(a)-(d) is for the shear stress $P_{12}=p_{12} /(\mu b / H)$ and Fig. 10(a)-(d) is for the shear 


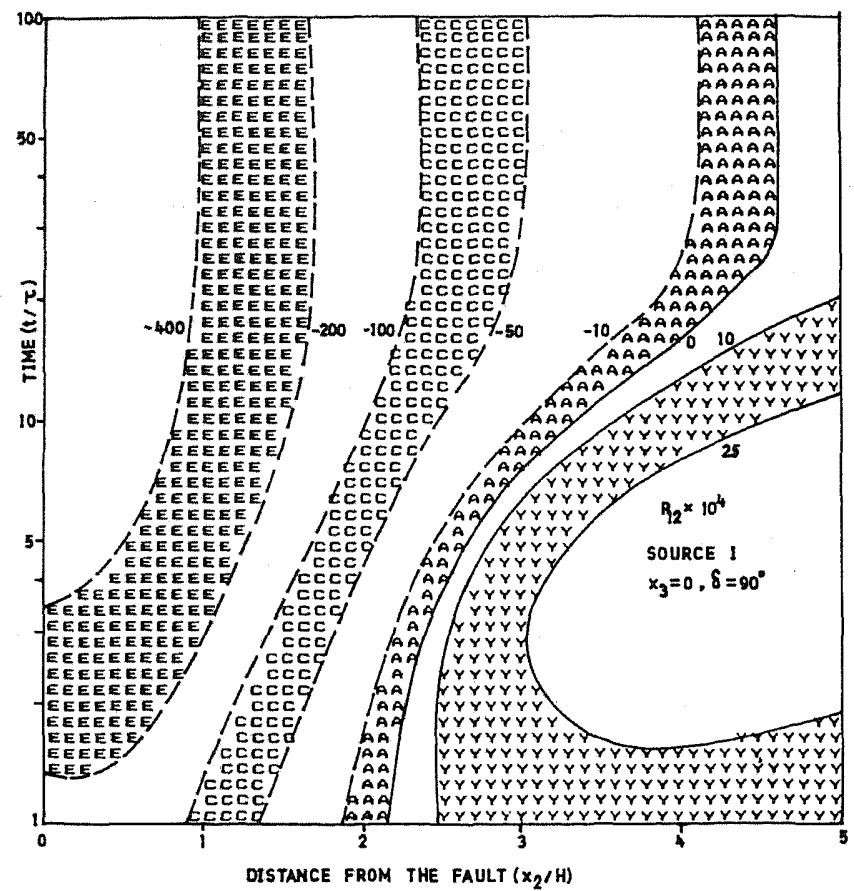

(a)

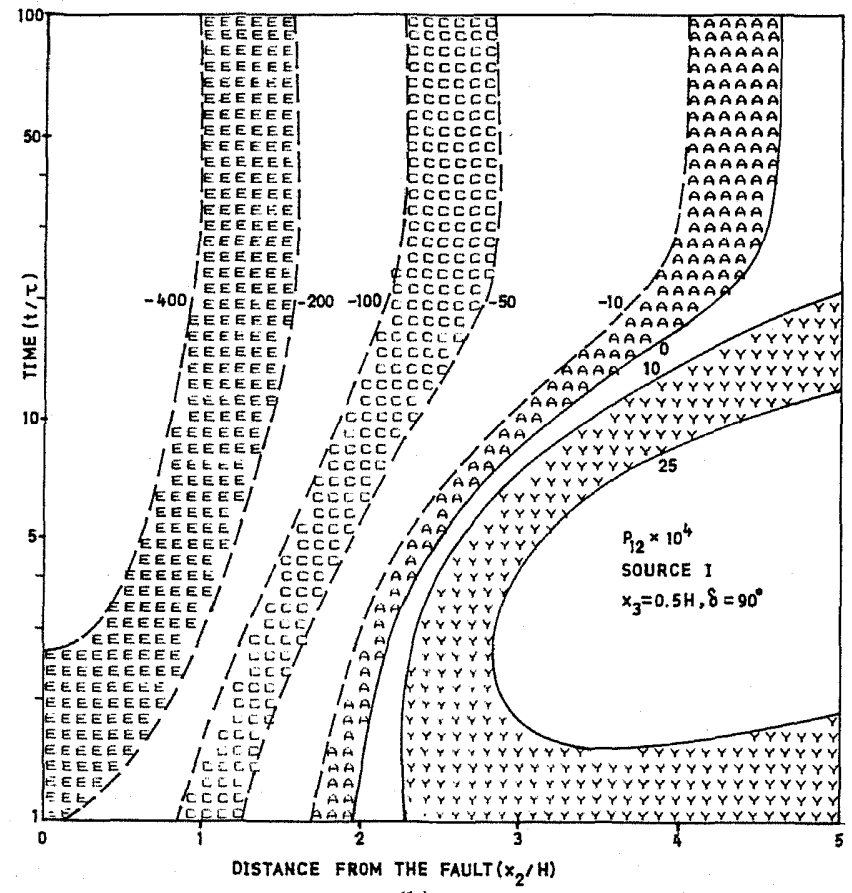

(b) 


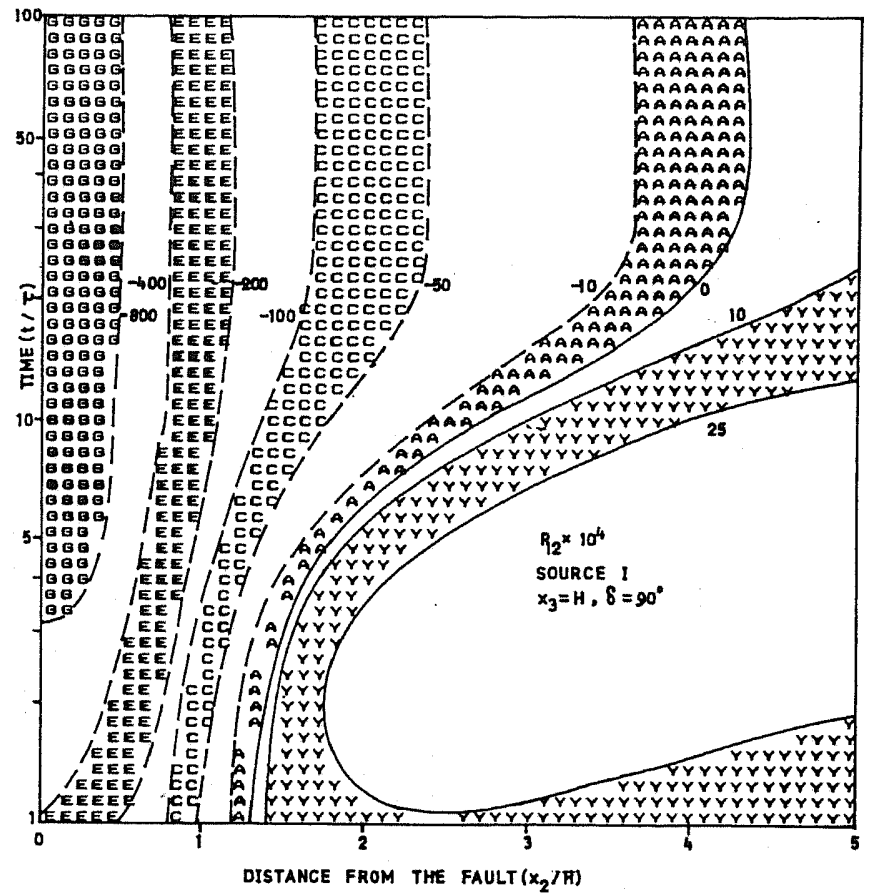

(c)

Fig. 12. Contours of constant postseismic shear stress $p_{12}$ parallel to the fault for (a) $x_{3}=0$, (b) $x_{3}=H / 2$, (c) $x_{3}=H . p_{12}$ is measured in units of $(\mu b / H) \times 10^{-4}$.

stress $P_{13}=p_{13} /(\mu b / H)$. From these figures, we notice that the location of the fault in the elastic layer has only a marginal effect on the deformation field in the substratum.

To study the spatial and temporal dependence of the postseismic deformation, contour maps of constant postseismic displacement and shear stress on the distance-time grid have been obtained. Figure 11(a) shows the contours of constant postseismic parallel surface displacement on the distance from the fault-time after the earthquake grid for a surface-breaking vertical fault of depth $H / 4$ (Source I). The displacement is measured in units of $b \times 10^{-3}$, distance in units of the lithospheric thickness $H$ and time in units of the asthenospheric relaxation time $\tau=\eta / 2 \mu_{2}$. Figure 11(b) and (c) is for the subsurface displacements. Contours of constant postseismic'shear stress $p_{12}$ are shown in Fig. 12(a) to (c). In these figures, the shear stress is measured in units of $(\mu b / H) \times 10^{-4}$, distance in units of $H$ and time in units of the relaxation time $\tau$. Figure 13(a) and (b) is for the subsurface postseismic shear stress $p_{13}$. While shear stress $p_{12}$ acts on planes parallel to the fault, shear stress $p_{13}$ acts on planes parallel to the Earth's surface. Figure 12(a) to (c) shows that as the point of observation in the layer moves away from the surface, the nodal line for the shear stress $p_{12}$ moves towards the fault. Furthermore, with the passage of time, the nodal line for $p_{12}$ moves away from the fault. The same is true for the nodal line for shear stress $p_{13}$. 
(a)

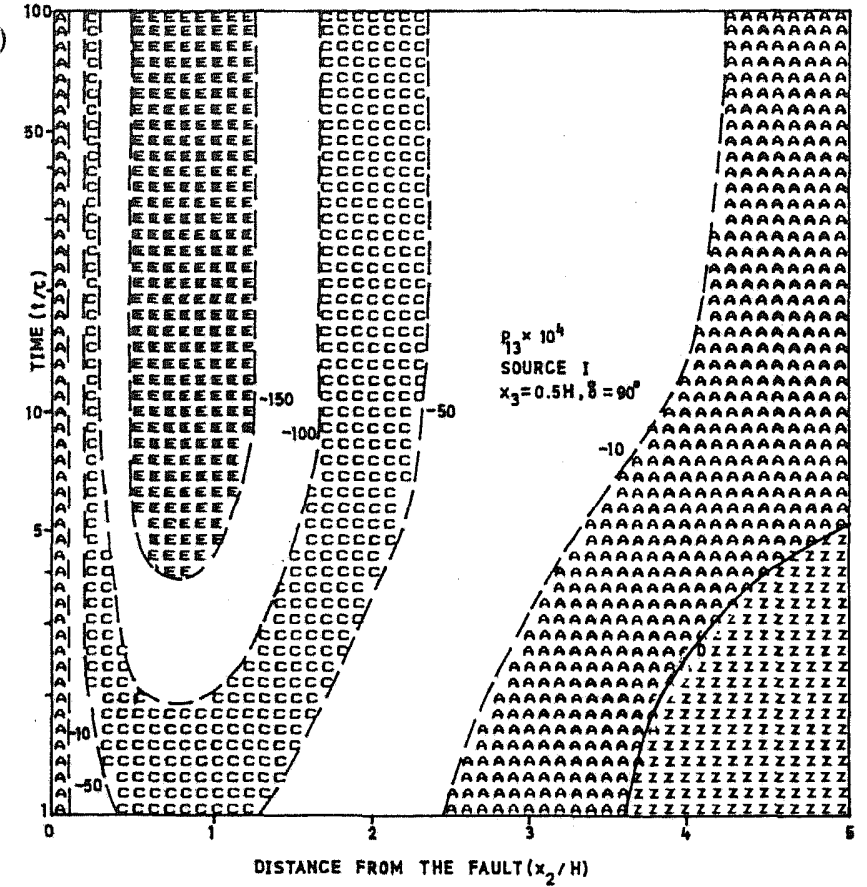

(b)

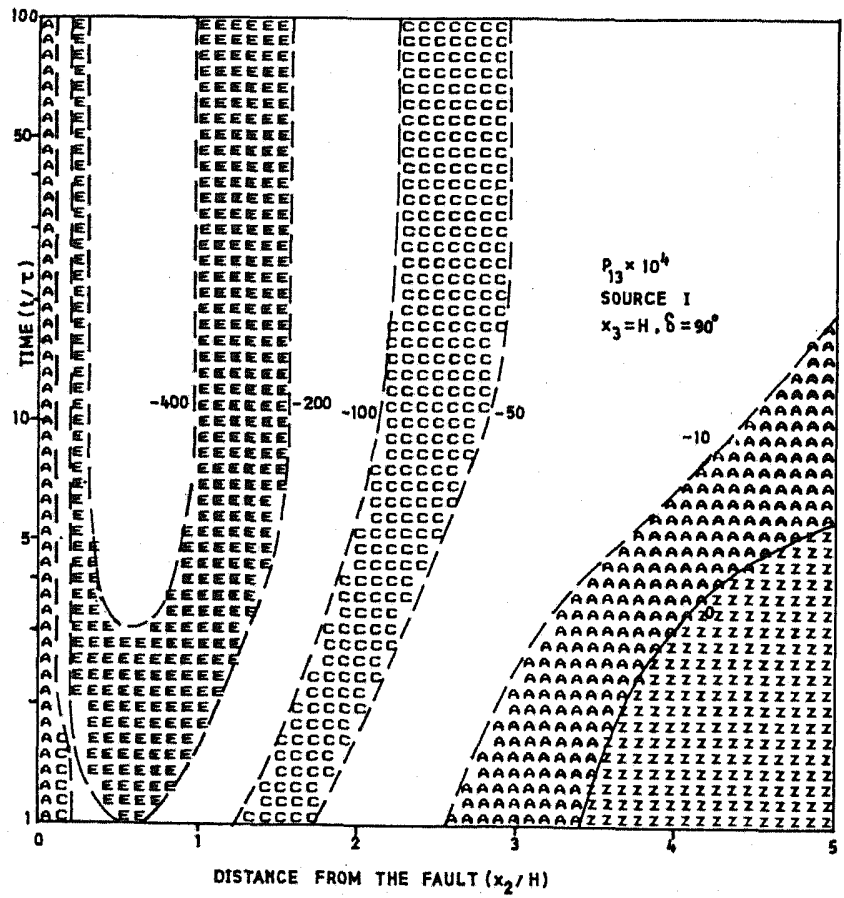

Fig. 13. Contours of constant postseismic shear stress $p_{13}$ parallel to the Earth's surface for (a) $x_{3}=H / 2$, (b) $x_{3}=H . p_{13}$ is measured in units of $(\mu b / H) \times 10^{-4}$. 


\section{Discussion}

We have studied strike-slip faulting in the lithosphere for a long fault of arbitrary dip and finite width. Although a 2-D model is an oversimplification of the physical system, such models are useful in gaining insight into the relationship among various fault parameters. Moreover, there are faults, the most classic example of which is the San Andreas fault, which is sufficiently long and shallow that the 2-D approximation may be used. Detailed numerical computations reveal that the deformation field depends greatly on the location of the fault and its dip. The field due to a surface-breaking fault is basically different from the field due to a deep fault. For a surface-breaking fault, the surface displacement parallel to the fault is discontinuous at the fault-trace, while for deep faults, it is continuous there. For a surface-breaking fault, the ratio of the absolute displacement of the hanging wall to that of the foot wall is a sensitive indication of the dip angle. Similarly, the degree of asymmetry of the surface displacement field depends upon the dip angle. The coseismic and postseismic displacements exhibit different behaviors. There are regions in which the coseismic and postseismic displacements (and stresses) are of different signs. The nodal lines for the postseismic displacement are fixed with respect to time. In contrast, the nodal lines for postseismic shear stresses move away from the fault with time after the earthquake.

The explicit analytical expressions giving the elastic as well as the viscoelastic solution involve infinite series. Therefore, a few remarks regarding their convergence are in order. From Eq. (7), we note that

$$
\begin{aligned}
(2 n H+ & \left.x_{3}-y_{3}\right) / V^{2}-\left(2 n H+x_{3}+y_{3}\right) / W^{2} \\
& =2 y_{3}\left[\left(2 n H+x_{3}\right)^{2}-y_{3}{ }^{2}-\left(x_{2}-y_{2}\right)^{2}\right] / V^{2} W^{2} \\
& =O\left(n^{-2}\right), \\
V^{-2} & =O\left(n^{-2}\right), \quad W^{-2}=O\left(n^{-2}\right),
\end{aligned}
$$

as $n \rightarrow \infty$. Moreover, $|r|<1$. Therefore, the infinite series appearing in the right-hand side of Eq. (6) converge very rapidly. Numerically, we have found that the first couple of terms in the infinite series are adequate for most practical purposes. Similar remarks are also applicable in the case of the other infinite series appearing in the elastic as well as the viscoelastic solution. In our computations, we have taken into consideration the first ten terms of the infinite series.

We are thankful to the Council of Scientific and Industrial Research, New Delhi and the National Board for Higher Mathematics, Bombay for financial support. We are also thankful to the referees for their helpful comments on the presentation of this paper.

\section{REFERENCES}

Bonafede, M., E. Boschi, and M. Dragoni, A dislocation model of microplate boundary ruptures in the presence of a viscoelastic asthenosphere, Geophys. J.R. Astron. Soc., 76, 515-529, 1984.

Cohen, S. C., Postseismic surface deformations due to lithospheric and asthenospheric viscoelasticity, Geophys. Res. Lett., 6, 129-131, 1979.

Vol. 42, No. 3, 1994 
Cohen, S. C., A multilayer model of time-dependent deformation following an earthquake on a strike-slip fault, J. Geophys. Res., 87, 5409-5421, 1982.

Dragoni, M. and D. A. Yuen, Global postseismic deformation in a stratified viscoelastic earth: effects on Chandler wobble excitation, J. Geophys. Res., 88, 2240-2250, 1983.

Dragoni, M., C. Magnanensi, and E. Boschi, The effect of faulting on the moment of inertia of a two-layer planet with Burgers-body mantle, Phys. Earth. Planet. Inter., 43, 223-235, 1986.

Erdélyi, A., Tables of Integral Transforms, Vol. 1, McGraw-Hill, New York, 1954.

Garg, N. R. and R. K. Sharma, Deformation of an elastic layer coupling in different ways to a base due to a very long vertical strike-slip dislocation, Proc. Indian Acad. Sci. (Earth Planet. Sci.), 101, 255-268, 1992.

Iwasaki, T., Quasi-static deformation due to a dislocation source in a Maxwellian viscoelastic earth model, J. Phys. Earth, 33, 21-43, 1985.

Iwasaki, T., Displacement, strain, and stress within a viscoelastic half-space due to a rectangular fault, J. Phys. Earth, 34, 371-396, 1986.

Iwasaki, T. and M. Matsu'ura, Quasi-static strains and tilts due to faulting in a layered half-space with an intervenient viscoelastic layer, J. Phys. Earth, 29, 499-518, 1981.

Lee, E. H., Stress analysis in viscoelastic bodies, Q. Appl. Math., 13, 183-190, 1955.

Matsu'ura, M. and T. Sato, A. dislocation model for the earthquake cycle at convergent plate boundaries, Geophys. J. Int., 96, 23-32, 1989.

Matsu'ura, M. and T. Tanimoto, Quasi-static deformations due to an inclined rectangular fault in a viscoelastic half-space, J. Phys. Earth, 28, 103-118, 1980.

Matsu'ura, M., T. Tanimoto, and T. Iwasaki, Quasi-static displacements due to faulting in a layered half-space with an intervenient viscoelastic layer, J. Phys. Earth, 29, 23-54, 1981.

Nur, A. and G. Mavko, Postseismic viscoelastic rebound, Science, 183, 204-206, 1974.

Pollitz, F. F., Postseismic relaxation theory on the spherical earth, Bull. Seismol. Soc. Am., 82, 422-453, 1992.

Pollitz, F. F. and I. S. Sacks, Modeling of postseismic relaxation following the great 1857 earthquake, Southern California, Bull. Seismol. Soc. Am., 82, 454-480, 1992.

Rosenman, M. and S. J. Singh, Quasi-static strains and tilts due to faulting in a viscoelastic half-space, Bull. Seismol. Soc. Am., 63, 1737-1752, 1973 a.

Rosenman, M. and S. J. Singh, Stress relaxation in a semi-infinite viscoelastic earth model, Bull. Seismol. Soc. Am., 63, 2145-2154, 1973 b.

Rundle, J. B., Viscoelastic crustal deformation by finite quasi-static sources, J. Geophys. Res., 83, 5937-5945, 1978.

Rundle, J. B. and D. D. Jackson, A three-dimensional viscoelastic model of a strike-slip fault, Geophys. J. R. Astron. Soc., 49, 575-591, 1977.

Rybicki, K., The elastic residual field of a very long strike-slip fault in the presence of a discontinuity, Bull. Seismol. Soc. Am., 61, 79-92, 1971.

Savage, J. C. and W. H. Prescott, Asthenosphere readjustment and the earthquake cycle, $J$. Geophys. Res., 83, 3369-3376, 1978.

Singh, S. J., Static deformation of a multilayered half-space by internal sources, J. Geophys. Res., 75, 3257-3263, 1970.

Singh, S. J. and N. R. Garg, On two-dimensional elastic dislocations in a multilayered half-space, Phys. Earth Planet. Inter., 40, 135-145, 1985.

Singh, S. J. and M. Rosenman, Quasi-static deformation of a viscoelastic half-space by a displacement dislocation, Phys. Earth Planet. Inter., 8, 87-101, 1974. 